\title{
Effective computation of matrix elements between polynomial basis functions
}

\author{
Igor N. Kozin $^{\mathrm{a}}$, Jonathan Tennyson ${ }^{\mathrm{b}}$, and Mark M. Law ${ }^{\mathrm{a}}$ \\ ${ }^{a}$ Department of Chemistry, University of Aberdeen, Meston Walk, Aberdeen AB24 \\ उUE, $U K$ \\ ${ }^{\mathrm{b}}$ Department of Physics and Astronomy, University College London, London \\ WC1E $6 B T, U K$
}

\begin{abstract}
Two methods of evaluating matrix elements of a function in a polynomial basis are considered: expansion method, where the function is expanded in the basis and the integrals are evaluated analytically, and numerical method, where the integration is performed using numerical quadrature. A reduced grid is proposed for the latter which makes use of the symmetry of the basis. Comparison of the two methods is presented in the context of evaluation of matrix elements in the non-direct product basis If high accuracy of all matrix elements is required then the expansion method is the best choice. If however the accuracy of high order matrix elements is not important, (as in variational ro-vibrational calculations where one is typically interested only in the lowest eigenstates) then the method based on the reduced grid offers sufficient accuracy and is much quicker than the expansion method.
\end{abstract}

Key words:

$P A C S$ :

In many physical applications the problem of effective evaluation of integrals of a multi-dimensional function often arises. For example, the typical situation in the calculation of molecular rotation-vibration spectra is evaluation of matrix elements which are integrals of the product of two general polynomial functions (basis functions) and an arbitrary function (molecular potential). Depending on the dimensionality and the number of matrix elements such 
calculations are often computationally very demanding. It is often the case that the properties of the polynomial functions are very well known and there are analytical formulae for the integrals of these functions. Reference [1] considered the case when the basis functions are a direct product of standard polynomials and advocated the expansion method, i.e., expansion of the potential in the basis and subsequent evaluation of the integrals analytically. In this paper, we propose a method of numerical integration on a reduced grid which makes use of the symmetry properties of the basis functions. Considering an example of a non-direct product basis we argue that our method of direct numerical integration can be very competitive to the expansion method and may even be a preferable choice.

Our starting point is a model for floppy four-atomic molecules as implemented in the new program WAVR4 [7]. The general description of the approach can be found elsewhere [2-5] and more details about the implementation in Ref. $[6,7]$. Of the six internal coordinates, the three radial coordinates are treated using the discrete variable representation (DVR) [9]. The DVR approximation for the potential reduces six dimensional integrals to three dimensional. The other three coordinates are the angles $\left(\theta_{1}, \theta_{2}, \varphi\right)$ which are represented by a non-direct product basis

$$
|j l k J K\rangle=\bar{P}_{j}^{|k-K|}\left(\theta_{1}\right) \bar{P}_{l}^{k}\left(\theta_{2}\right) \frac{\mathrm{e}^{i k \varphi}}{\sqrt{2 \pi}}|J K\rangle,
$$

where $J$ and $K$ are the usual rotational quantum numbers, $j$ and $l$ are angular momenta associated with internal coordinates, $k$ is projection of $l$ on the quantization axis, $\bar{P}_{l}^{k}$ are normalized associated Legendre functions, $|J K\rangle$ are symmetric top eigenfunctions. All the quantum numbers take only nonnegative integer values.

The straightforward approach is to use Gaussian quadrature [10,11]

$$
\int_{a}^{b} W(x) p_{m}(x) d x=\sum_{i=1}^{N} w_{i} p_{m}\left(x_{i}\right)
$$

where $W(x)$ is weighting function and $w_{i}$ are weights. The grid points $x_{i}$ are the roots of the polynomial $p_{N}(x)$ of order $N$ and the quadrature is exact for all polynomials $p_{m}(x)$ to degree $(2 N-1)$. So if we have Legendre type basis functions $P_{l}(x)$ up to order $l_{\max }$, we need $N=l_{\max }+1$ or more quadrature points to compute the integrals

$$
\int_{-1}^{1} P_{l_{1}}(x) f(x) P_{l_{2}}(x) d x \approx \sum_{i=1}^{N} P_{l_{1}}\left(x_{i}\right) f\left(x_{i}\right) P_{l_{2}}\left(x_{i}\right) .
$$


For the basis function given in Eq.(1) we can use Legendre quadrature for associated Legendre functions (in $\theta_{1}$ and $\theta_{2}$ ) and equidistant (Fourier) grid for $\exp i k \varphi$ functions (in $\varphi$ ). Associated Legendre functions require double the number of quadrature points which otherwise would be sufficient for Legendre functions of the same order. If we characterise the size of our basis using $\left(j_{\max }, l_{\max }, k_{\max }\right)$ we can define a minimal grid size $\left(2 j_{\max }+1\right) \times\left(2 l_{\max }+1\right) \times$ $\left(2 k_{\max }+1\right)$, i.e. the minimal grid on which our basis is still orthogonal. It is easy to see that evaluation of all matrix elements using multi-dimensional quadrature can be a bottle-neck.

One way to reduce the computational cost is to use expansion of integrated function [1]. In our case we can use the following expansion

$$
V\left(\theta_{1}, \theta_{2}, \varphi\right)=\sum_{j l k} a_{j l k} \bar{P}_{j}^{k}\left(\theta_{1}\right) \bar{P}_{l}^{k}\left(\theta_{2}\right) \frac{\mathrm{e}^{i k \varphi}}{\sqrt{2 \pi}}
$$

because the potential function of a molecule is rotationally invariant, i.e. only $J=K=0$ basis functions are required in the expanded potential. Reference [8] provides an elegant way of expressing the matrix elements of the expanded potential Eq. (4) through integrals of products of spherical harmonics. The latter can be evaluated analytically through $3 J$-symbols. Here, we take a shortcut and simply define

$$
\int \bar{P}_{l_{1}}^{k_{1}}(x) \bar{P}_{l_{2}}^{k_{2}}(x) \bar{P}_{l_{3}}^{k_{3}}(x) d x=A\left(l_{1}, l_{2}, l_{3} ; k_{1}, k_{2}, k_{3}\right)
$$

where

$$
\begin{aligned}
& A\left(l_{1}, l_{2}, l_{3} ; k_{1}, k_{2}, k_{3}\right)=(-1)^{\left(m_{1}-k_{1}+m_{2}-k_{2}+m_{3}-k_{3}\right) / 2} \\
& \times \sqrt{\left(2 l_{1}+1\right)\left(l_{2}+1 / 2\right)\left(2 l_{3}+1\right)}\left(\begin{array}{ccc}
l_{1} & l_{2} & l_{3} \\
0 & 0 & 0
\end{array}\right)\left(\begin{array}{ccc}
l_{1} & l_{2} & l_{3} \\
m_{1} & m_{2} & m_{3}
\end{array}\right) .
\end{aligned}
$$

Since $k_{i} \geq 0$ by definition, $m_{i}$ are chosen so that $\left|m_{i}\right|=k_{i}$ and the $3 J$-symbol rule holds: $m_{1}+m_{2}+m_{3}=0$. A second $3 J$-symbol rule must be satisfied too: $\left|l_{1}-l_{3}\right| \leq l_{2} \leq l_{1}+l_{2}$.

Then the matrix elements of the potential function are

$$
\begin{aligned}
& \left\langle j_{1} l_{1} k_{1} J K|V| j_{3} l_{3} k_{3} J K\right\rangle=\frac{1}{\sqrt{2 \pi}} \\
& \times \sum_{j_{2} l_{2} k_{2}} a_{j_{2} l_{2} k_{2}} A\left(j_{1}, j_{2}, j_{3} ;\left|K-k_{1}\right|, k_{2},\left|K-k_{3}\right|\right) A\left(l_{1}, l_{2}, l_{3} ; k_{1}, k_{2}, k_{3}\right)
\end{aligned}
$$


The $3 J$-symbol rules provide the upper limit for the expansion size: twice the maximum for each quantum number used in the basis. However the sum in Eq. (7) is smaller than the sum over the minimal grid for the same basis not only because of the non-direct nature of our basis but also because many terms in Eq. (7) are zero due to $3 J$-symbol rules (see Ref. [1] for more details).

In spite of the attractions of the expansion method we have found it valuable to reconsider direct numerical integration. It turns out we can do much better if we use properties of our basis. It is frequently the case that the integration interval is symmetric and the basis functions can be symmetric or antisymmetric on the interval. Consider again the integral (3). The function $f(x)$ may be expressed as a sum of symmetric $f_{s}(x)=f_{s}(-x)$ and anti-symmetric $f_{a}(x)=-f_{a}(-x)$ parts. If the product $P_{l_{1}}(x) P_{l_{2}}(x)$ is symmetric we need to integrate only the symmetric part of $f(x)$ because the anti-symmetric part gives zero. This allows us to sum only over positive quadrature points and then double the sum. The analogous argument applies if $P_{l_{1}}(x) P_{l_{2}}(x)$ is antisymmetric. Assuming $N$ is even we can write

$$
\int_{-1}^{1} P_{l_{1}}(x) f(x) P_{l_{2}}(x) d x \approx\left[\begin{array}{l}
\sum_{i=1}^{N / 2} P_{l_{1}}\left(x_{i}\right) f_{s}\left(x_{i}\right) P_{l_{2}}\left(x_{i}\right) \text { if } l_{1}+l_{2} \text { is even } \\
\sum_{i=1}^{N / 2} P_{l_{1}}\left(x_{i}\right) f_{a}\left(x_{i}\right) P_{l_{2}}\left(x_{i}\right) \text { if } l_{1}+l_{2} \text { is odd }
\end{array}\right.
$$

Therefore the knowledge of the symmetry properties of our basis can be used to reduce the computational cost.

Note, that the symmetry properties of the basis may have nothing to do with the symmetry of the molecule in question. Even if the symmetry $x \rightarrow-x$ above is absent in the molecular system one can still get a twofold computational gain due to the decomposition of the function to be integrated. If symmetry is present in the molecular system then $f_{a}(x)=0$ and the whole problem splits in two: one involving only a symmetric basis and another involving an antisymmetric basis. In this case, it is usual to use the symmetry of coordinate space and reduce the number of quadrature points. This reduction can be viewed as a sub-case of the more general case of a system without symmetry and therefore we suggest always implementing the more general algorithm described above.

There are more elaborate versions of reducing the cost of integration like Fast Fourier Transformation (FFT) for cos/sin functions. Recently a combination of FFT with recursive methods for associated Legendre functions was used to compute expansions of spherical harmonics $[12,13]$. However our simple algorithm offers a reasonable compromise between algorithm complexity and considerable computational savings. It is very easy to implement and generalise in many dimensions where it gives $2^{\mathrm{dim}}$ saving. Thus for the basis (1) the minimal grid on which the basis is still orthogonal is $\left(j_{\max }+1\right) \times\left(l_{\max }+1\right) \times\left(k_{\max }+1\right)$ 
and therefore represent eightfold saving [14]. It turns out that using the reduced grid is very competitive compared to the expansion method. First of all the analytical integration requires $3 J$-symbols. Unfortunately it is usually not practical to precompute and store them because the required storage size is too big. Therefore they need to be computed every time they are needed. Secondly the implementation of analytical integration requires some logic based on the $3 J$-symbol rules but this typically degrades processor performance. On the other hand for the numerical integration we need only to precompute the basis functions and potential on the grid, and perform a direct multiplication-summation. The memory requirements are small (for example, for $j_{\max }=l_{\max }=k_{\max }=30$ one needs less than 1MB) and the method performs very well. Our tests showed that the implementation in WAVR4 performs the numerical integration on the minimal grid much quicker than the expansion method (even not including the expansion itself). The accuracy provided by the minimal grid was found to be acceptable because in variational calculations we are typically interested in the lowest eigenfunctions and therefore some inaccuracy of high matrix elements is not critical. However one may want to use a slightly bigger reduced grid to achieve a suitable compromise between speed and accuracy. For example, it was suggested to use $\left(n_{\max }+4\right)$ points instead of $\left(n_{\max }+1\right)$ for polynomials of order $n_{\max }[15]$. Only when high accuracy is vital the use of an expansion becomes the preferable choice because comparable accuracy using numerical integration becomes more expensive. Both the expansion and numerical options are implemented

in WAVR4 and the user may choose the optimal strategy for the problem of interest.

\section{References}

[1] J. Tennyson, Comp. Phys. Comm., 38, 39 (1985).

[2] X. Chapuisat and C. Iung, Phys. Rev. A 45, 6217 (1992).

[3] F. Gatti, C. Iung, M. Menou, Y. Justum, A. Nauts, and X. Chapuisat, J. Chem. Phys. 108, 8804 (1998).

[4] C. Iung, F. Gatti, A. Viel, and X. Chapuisat, Phys. Chem. Chem. Phys. 1, 3377 (1999).

[5] M. Mladenović, J. Chem. Phys. 112, 1070 (2000).

[6] I. N. Kozin, M. M. Law, J. M. Hutson and J. Tennyson, J. Chem. Phys. 118, 4896 (2003).

[7] I. N. Kozin, M. M. Law, J. Tennyson and J. M. Hutson, in preparation.

[8] J. Tennyson and A. van der Avoird, J. Chem. Phys. 77, 5664 (1982). 
[9] J. C. Light, I. P. Hamilton and J. V. Lill, J. Chem. Phys. 92, 1400 (1985).

[10] A. H. Stroud, and D. Secrest, Gaussian Quadrature Formulas. Englewood Cliffs, NJ: Prentice-Hall, 1966.

[11] W. H. Press, B. P. Flannery, S. A. Teukolsky, and W. T. Vetterling, Numerical Recipes in FORTRAN: The Art of Scientific Computing, 2nd ed. Cambridge, England: Cambridge University Press, 1992.

[12] J. R. Driscoll and D. M. Healy, Jr., Adv. Appl. Math. 15, 202 (1994).

[13] V. Lesur and D. Gubbins, Geophys. J. Int. 139, 547 (1999).

[14] In practice our basis (1) is adapted to inversion symmetry which takes $\varphi \rightarrow$ $2 \pi-\varphi$ (see Ref. [5,6]). Therefore our expansion is also parity adapted. It is straightforward to apply Eq. (5) again and show that the matrix elements have a similar form. Respectively our grid in $\varphi$ is halved: the non-reduced minimal grid has $\left(k_{\max }+1\right)$ points; the reduced one has $\left(k_{\max }+1\right) / 2$ points if $k_{\max }$ is odd and $k_{\max } / 2+1$ if $k_{\max }$ is even.

[15] J. Tennyson and B. T. Sutcliffe, J. Chem. Phys. 77, 4061 (1982). 